\title{
Transformer health index prediction using feedforward neural network according to scoring and ranking method
}

\author{
Nur Ashida Salim ${ }^{1 *}$, Jas ronita Jasni ${ }^{2}$, Hasmaini Mohamad ${ }^{1}$ and Zuhaila Mat Yas in ${ }^{1}$ \\ Faculty of Electrical Engineering, Universiti Teknologi MARA, Shah Alam, Selangor, Malaysia ${ }^{1}$ \\ Advanced Lightning, Power \& Energy Research, Faculty of Engineering, Universiti Putra Malaysia ${ }^{2}$
}

Received: 08-October-2020; Revised: 11-February-2021; Accepted: 14-February-2021

(C)2021 Nur Ashida Salim et al. This is an open access article distributed under the Creative Commons Attribution (CC BY) License, which permits unrestricted use, distribution, and reproduction in any medium, provided the original work is properly cited.

\begin{abstract}
This paper presents a transformer health index prediction applying feedforward neural network (FFNN) according to scoring and ranking method. Power transformer is an important asset of a power system where the function is to convert the level of electrical power and transfer it to the consumer. Outage in transmission line that is caused by the power transformer might lead to the interruption of power supply. Transformer asset management is vital to monitor the operation of transformers in the system to prevent failure. The technique in performing asset management of the transformer is health index (HI). Therefore, this paper presents the assessment of transformer HI by applying artificial neural network (ANN). The FFNN training algorithms proposed in this research to predict the transformer HI include Levenberg-Marquardt (LM), quasi-Newton backpropagation (QNBP), and scaled conjugate gradient (SCG). The HI values obtained from these FFNN techniques were compared to the scoring and ranking method to validate the proposed technique. The performance of the proposed ANN was assessed according to the correlation coefficient and mean square error (MSE). According to the findings obtained, the transformer HI can be successfully predicted by applying different training algorithm of ANN. LM, QNBP and SCG proposed in this research could identify whether the transformer condition is very good, good, fair or poor. The ANN proposed in this research also has been verified with the ranking and scoring method where it produces similar identification of the transformer health index. According to the HI, advance action could be initiated whether to perform upgrade, maintenance, replacement, monitoring, repair, and contingency control of the transformer.
\end{abstract}

\section{Keywords}

Feedforward neural network, Levenberg-marquardt, Quasi-newton backpropagation, Scaled conjugate gradient, Transformer health index.

\section{Introduction}

Power transformers have become one of the most vital assets in the energy transmission and power system. If any transformers fail to function properly, it will result in electrical supply interruptions and affect the reliability of the system. Power transformer is the most valuable and critical asset for utility companies and its condition needs to be monitored with proper assessments. Nearly $60 \%$ of the total investment is allocated for power transformers, and it becomes the highest value equipment in high-voltage substations [1]. Due to the lack of proper maintenance, many transformers fail to function and operate before they reach their rated lifetime.

\footnotetext{
*Author for correspondence
}

Transformer life expectancy gradually decreases due to oxidation, humidity and temperature, acidity and pollution of oil insulation, seal, and deterioration of the gasket [2].

If the maintenance and assessment could not detect the presence of failure in the transformer, it may cause catastrophic failure which will affect the electric power system. As the demand on high system reliability increases, many electrical utilities have to concentrate more on the effectiveness of asset management. Performing an asset management would be the best way to overcome these problems because it could increase the reliability of the asset and reduce the main capital expenditure to either overhaul or change the assets. Asset management is known as the procedure of exploiting the return on 
investment of equipment by providing the required level of performance and reducing cost over the life cycle of the system [3]. Health index (HI) is used to measure the findings of site and laboratory testing, field inspections, and functioning observations into a quantitative index and objective, providing the overall health of the assets. The asset $\mathrm{HI}$ is a powerful tool that can recognize investment requirements, prioritize investments, and organize assets into maintenance and capital programmes [4].

Generally, many techniques and assessment models have been used by researchers to perform the asset management. Pandey and Sonwane [5] presented about reliability centered maintenance (RCM) which includes many intermediate techniques such as failure mode and effect analysis (FMEA) and consequences of failure analysis (COFA). The research aims to attain cost effectiveness by monitoring maintenance routine and improving consistency. BlockSim Software was used for reliability analysis which can be a series, parallel model, or a combination of both transformers. Phadungthin and Haema in [6] used the scoring and weighting methods for transformer risk assessment. In scoring, the conditions are scored according to the planned limit. For example, the diagnostic tests for condition assessments are divided into five scorings which are 1 (very good), 2 (good), 3 (moderate), 4 (poor), and 5 (very poor). Weighting is referred as the items are weighted according to their importance. The scores and weight of each criterion are determined by specialists in the utilities. The findings for both assessments of the transformer are plotted in the risk model to achieve the proper bushing maintenance. A. Abu-Elanien et al. [7] suggested a method of finding a health condition transformer applying HI. Transformer HI was determined by a feedforward artificial neural network (FFANN) applying the actual measurement of the working transformer. In the study, FFANN was used to investigate the relationships between 11 data sets and the HI of each transformer with the support of the health indices given by the asset management health assessment (AMHA). Besides, W. Wattakapaiboon et al. presented a new improved technique of conventional $\mathrm{HI}$ table to evaluate the state of the transformer [8]. The scores gained from the conventional $\mathrm{HI}$ tables and new advanced $\mathrm{HI}$ tables were compared to measure the performance of the new HI tables. According to the findings, the difference (mean value of all case studies) between the advanced $\mathrm{HI}$ schedule and the conventional $\mathrm{HI}$ method was only $7 \%$. Research paper [9] presents the capability of a Malaysian utility company, Tenaga Nasional Berhad (TNB) in handling condition assessments and life management programmes on power transformers through a diagnostic method and calculation of transformer HI. There are three main stages called as Tier 1, Tier 2, and Tier 3 . The failure mode and critical analysis (FMECA) was selected for the condition-based maintenance. For condition assessment, transformer $\mathrm{HI}$ is used by the power system utility to inspect the condition of the transformer and decide whether to perform upgrade, maintenance, replacement, monitoring, repair, or contingency control [10]. F. Scatiggio et al. presented a new model of periodic test evidence (dissolved gas analysis [DGA], furans, acidity, inductance, frequency dielectric spectroscopy) that combines with the koranic properties of a substation [11]. The first parameter is static depending on the koranic level (frequency of light) on the location, the substation layout, and repetition of catastrophic events on the site. The next parameter depends entirely on the transformer and must be considered dynamic. The HI of the transformer is created from the combination of two parameters. Each transformer is divided according to the $\mathrm{HI}$ range according to the probability of failure and degrees of strength in terms of electrical, thermal, mechanical, and oil conditions.

According to the detailed review of the subject matter, the main objective of this paper is to model an artificial neural network (ANN) to evaluate the HI of a transformer. The ANN learns by examples which is self-learning, self-studying, and self-adaption; it can be trained with known data sets for acquiring knowledge and experience [12]. The feedforward neural network (FFNN) was applied in this research, and it comprises of input, output, and hidden layers. The ANN was inspired by the biological neural network in human brain that serves as an information processing model [13]. It has a significant skill to get the meaning from complex or inaccurate data. It is used to extract the trend or pattern which is difficult to be solved by humans and computers. This study proposed three types of ANN training algorithm which are Levenberg-Marquadt (LM), quasi-Newton backpropagation (QNBP), and scaled conjugate gradient (SCG) to predict the transformer HI. These three training algorithms are the novelty of this paper where none of the previous researchers have considered. The validation of the proposed model was done by comparing the proposed method with the scoring and ranking method. 
Nur Ashida Salim et al.

\section{Material and methods}

This section explains the methodology of transformer health assessment proposed in this research. First, the proposed technique of $\mathrm{ANN}$ is described in detail. The three ANN training algorithms which are LM, QNBP, and SCG are explained in this section. The scoring and ranking method is also explained. The explanation and implementation of the proposed method is limit to data and parameters obtained for this case study where it might differ from other types of transformer.

\subsection{FFNN using LM training method}

In this paper, FFNN applying LM training method was proposed to predict the HI of a transformer. The neural network comprises of several layers such as input, hidden, and output layers. The neural network will learn the relationship between the input and target to produce the predicted output. In ANN, the learning rate, momentum rate, and number of hidden layer help in accelerating the convergence of ANN model, speeding up the training process, and reducing the error between the targeted and predicted outputs [14]. The proposed FFNN structure with input, hidden, and output layers is shown in Figure 1. This is the system configuration that will be used throught this paper.

Normally, the range of learning rate and momentum rate is between 0 to 1 . The accuracy of the prediction was evaluated according to the mean square error (MSE) and mean absolute percentage error (MAPE), which can be obtained applying Equations (1) and (2), respectively:

$M S E=\frac{1}{N} \sum \frac{\left|P_{Q}-P_{P}\right|}{P_{Q}}$

where,

$N=$ total number of data

$P_{Q}=$ target result

$P_{p}=$ actual result

$M A P E=\frac{1}{T} \sum_{i=1}^{T} \frac{\mid \text { Hi.target }- \text { Hi.predict } \mid}{\text { Hi.target }} \times 100 \%$

Where,

$T=$ total number of data

Hi. target $=\mathrm{HI}$ target result

Hi. predict $=\mathrm{HI}$ predict result

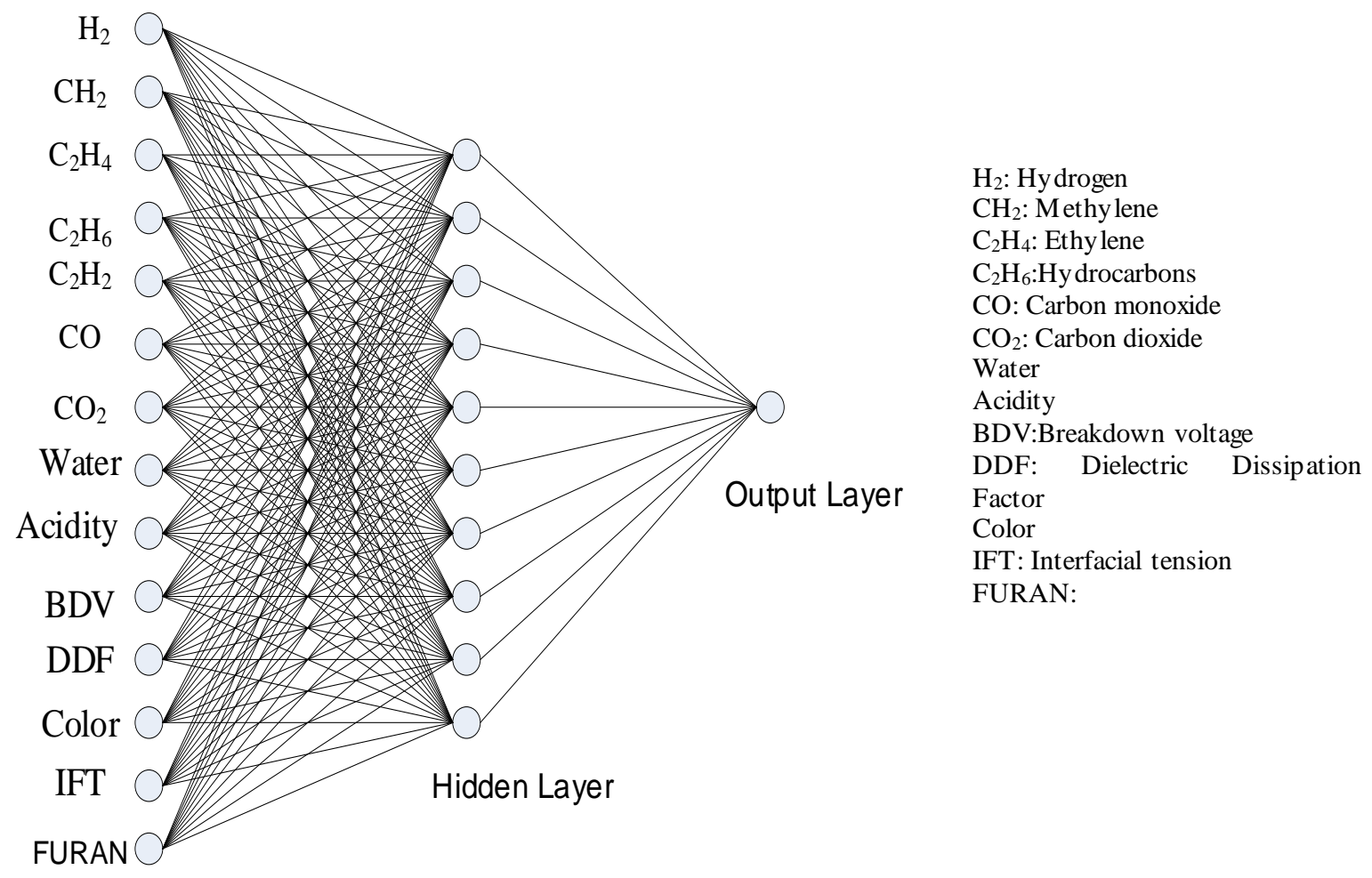

Input layer

Figure 1 Structure of proposed FFNN 
The LM training algorithm is one of the algorithms used for fitting problems in ANN trainings. The algorithm has a faster convergence and fewer iterations than other training algorithms. It is also relatively stable. This algorithm is the most suitable for heuristic techniques and known as the most accurate in reducing errors [14]. This algorithm can minimize the error [15], therefore it can be obtained applying Equations (3) and (4):

$x^{*} \in E^{N}$

Where,

$x^{*}=$ contained among the zeros of $\frac{1}{2} \nabla \emptyset(x)=$ $J(x)^{T} F(x)$

$\nabla \emptyset(x)=$ gradient of $\phi$

$J(x)=$ Jacobian matrix of $F$

$\emptyset(x)=\|F(x)\|_{2}^{2}=\sum_{i-1}^{M}|f i(x)|^{2}$

Where,

$F(x)=$ residual vector obtained from dataset by a nonlinear form

The ANN comprises of training and testing processes. In this study, the parameters include $70 \%$ training data and $30 \%$ testing data. The steps for the training process are described as follows:

a) The input and output parameters of the training are identified.

b) A trial and error method are implemented with the ratio of 70:30 training data.

c) The ANN configuration is designed.

d) The value of epochs, goal, learning rate, momentum rate, and hidden layer are set.

e) The training algorithm of LM backpropagation is set.

f) The ANN is executed, and the findings are analyzed and compared to the convergence result according to MSE and regression $(R)$. If the results did not converge, the heuristic method is used, the value of training parameter is modified, and the ANN is retrained.

g) All the outputs are saved for testing process.

Next, the testing process as described below was conducted after the training process of ANN was done.

a) The testing input and output data are identified.

b) The saved network from the training process is loaded.

c) The testing simulation process is executed.

d) The convergence of the result is compared according to the MSE and $R$.

e) If the result still does not converge, the network is retrained and the training procedure from the previous step is repeated.

f) All results are saved.

\subsection{FFNN using QNBP training method}

The QNBP training algorithm was used to complete the model. It has a high accuracy rate; thus, it has faster convergence speed [16]. The computation of iteration was obtained as in Equation (5) [17], where the Hessian matrix was used for the performance of the iteration. Since computing the iteration for FFNN is time consuming, the QNBP method was proposed as in Equation 5 [18]:

$X_{k+1}=X_{k}-H_{k}^{-1} g_{k}$

Where,

$X_{k} \quad=$ iteration $k$

$H_{k}{ }^{-1} g_{k}=$ Newton's training direction

The performance of the training process was determined according to the minimum error in MSE andMAPE that can be obtained by using Equations (1) and (2), respectively. ANN is a system that mimics the neural behavior in the human body. It contains perceptron or neuron units where its adjusted weight for predicting response is according to given input features. Creating the ANN system is comparable with the human learning process, where it should take more training before the testing process. According to the given input and desired output, the neuron or perceptron units learned by adjusting the interrelated neural weights. The learning process of FFNN determines the weight for connecting neurons with the inputs and outputs [19]. The procedure for the training applying QNBP is explained in the steps as follows:

a) The input and output parameters of the training are obtained.

b) The QNBP configuration is designed. The number of epochs, goals, momentum rate, learning rate, and number of hidden layers of the network related to the training algorithm are set.

c) Try and error method is used to get the best training parameter.

d) The results are analyzed from the FFNN-QNBP model. The result from the training and testing processes are compared according to the lowest MSE and the highest $R$ applying Equations (1) and (2), respectively. If the results did not converge, the value of training parameter is modified and the FFNN-QNBP model is retrained.

e) The best network is saved. The HI value of FFNNQNBP method is compared to that of the scoring and ranking method.

\subsection{FFNN using SCG training method}

The SCG was combined with the line search strategy for fast speed convergence as proposed by Leonard and Kramer. Mostly, the training algorithms used 
learning rate to update the weight and threshold. The SCG algorithm was updated automatically applying its own steps [20]. The search direction (Po) of the algorithm was started from the steepest descent direction (go), as shown in Equation (6).

$P o=-g_{o}$

The weight and threshold value were obtained applying Equation (7):

$X_{k+1}=X_{k}-\alpha_{k}+P_{k}$

Where,

$P_{\mathrm{o}}=$ search direction

$g_{\mathrm{o}}=$ steepest descent direction

$X=$ weight and threshold value

$\alpha=$ decrease the gradient in the search direction

The procedure for the training applying the SCG is explained in the steps as follows:

a) The input and output parameters of the training are obtained.

b) The SCG configuration is designed. The number of epochs, goals, momentum rate, learning rate, and number of hidden layers of the network that related to the training algorithm are set.

c) The try and error method is used to obtain the best training parameter.

d) The results from the FFNN-SCG model are analyzed. The results between the training and testing process are compared according to the lowest MSE and the highest R applying Equations (1) and (2), respectively. If the results did not converge, the value of training parameter is modified and the FFNN-SCG model is retrained.

e) The best network is saved. The HI value of FFNNSCG method is compared with that of the scoring and ranking method.
2.4Scoring and ranking method

The scoring and ranking method is a conventional technique to calculate the final $\mathrm{HI}$ value of a power transformer [21]. The input parameter for this method comprises of the outcomes of functioning observation, field assessments, and site and laboratory testing [10]. Next, the results are transformed to a quantitative index which indicate the general state of the transformer. Due to data limitation, only three parameters were considered in this study: oil quality, dissolved gasses in oil, and furans. The DGA technique was used to identify incipient faults which may happen in transformers such as partial discharge, thermal error, and electrical damage. In this study, seven gases were considered which are hydrogen $\left(\mathrm{H}_{2}\right)$, methane $\left(\mathrm{CH}_{4}\right)$, ethane $\left(\mathrm{C}_{2} \mathrm{H}_{6}\right)$, ethylene $\left(\mathrm{C}_{2} \mathrm{H}_{4}\right)$, acetylene $\left(\mathrm{C}_{2} \mathrm{H}_{2}\right)$, carbon monoxide $(\mathrm{CO})$, and carbon dioxide $\left(\mathrm{CO}_{2}\right)$. The oil quality test results represent the quality of oil and the parameters such as dielectric strength (BDV), dielectric dissipation factor (DDF), acidity, moisture, color, and oil's interfacial tension (IFT) were included. First, the scoring and weighting factor was determined for each parameters according to the corresponding ranges in [22]. The oil quality factor (OQAF) and dissolved gas analysis factor (DGAF) can be determined by applying Equation (8).

$D G A F / O Q A F=\frac{\sum_{i=1}^{n}\left(S_{i} \times W F_{i}\right)}{\sum_{i=1}^{n} W F_{i}}$

Where $S_{i}$ is the score of each parameter, $W F_{i}$ is the proper weighting factor for each condition parameter, and $n$ is the number of parameters in each factor. Next, the rating codes for DGAF, OQAF, and furans were determined according to the ranges in $[23,24]$ as shown in Tables 1, 2, and 3, respectively. These tables indicate the standard rating codes of the three parameters mentioned.

Table 1 Transformer rating code for DGAF

\begin{tabular}{lll}
\hline Rating Code & Conditions & Description \\
\hline A & Good & DGAF $<1.2$ \\
\hline B & Acceptable & $1.2 \leq$ DGAF $<1.5$ \\
\hline C & Need Caution & $1.5 \leq \mathrm{DGAF}<2$ \\
\hline D & Poor & $2 \leq \mathrm{DGAF}<3$ \\
\hline $\mathrm{E}$ & Very Poor & DGAF $\geq 3$ \\
\hline
\end{tabular}

Table 2 Transformer rating code for OQAF

\begin{tabular}{lll}
\hline Rating code & Conditions & Description \\
\hline A & Good & OQAF $<1.2$ \\
\hline B & Acceptable & $1.2 \leq \mathrm{OQAF}<1.5$ \\
\hline C & Need Caution & $1.5 \leq \mathrm{OQAF}<2$ \\
\hline $\mathrm{D}$ & Poor & $2 \leq \mathrm{OQAF}<3$ \\
\hline $\mathrm{E}$ & Very Poor & OQAF $\geq 3$ \\
\hline
\end{tabular}


Table 3 Furfural test rating

\begin{tabular}{ll}
\hline Rating code & Furaldehyde [ppm] \\
\hline A & $0-0.1$ \\
\hline B & $0.1-0.25$ \\
\hline C & $0.25-0.5$ \\
\hline D & $0.5-1.0$ \\
\hline E & more than 1.0 \\
\hline
\end{tabular}

The rating codes (A, B, C, D, and E) for each parameter was converted to a factor between 4 and 0 [25]. Then, the final HI was obtained by applying Equation (9).

Final $H I=\frac{\sum_{j=1}^{n}\left(K_{j} \times H I F_{j} j\right)}{\sum_{j=1}^{n} 4 K_{j}} \times 100 \%$
Where $H I F_{j}$ is the rating code for each parameter and $K_{j}$ is the weighting factor for the parameter. The condition of the transformer was determined by the value of final HI. The range is between $100 \%$ means very good condition to $0 \%$ means very poor condition. Table 4 shows the $\mathrm{HI}$ and expected lifetime of the transformer [26].

Table 4 Transformer $\mathrm{HI}$ and expected lifetime

\begin{tabular}{llll}
\hline Final HI (\%) & Condition & Expected lifetime & Requirement \\
\hline $86-100$ & Very Good & $\geq 15$ years & Regular Maintenance \\
\hline $71-85$ & Good & $\geq 10$ years & Regular Maintenance \\
\hline $51-70$ & Fair & from 3 to10 years & Increase Diagnostic Testing \\
\hline $31-50$ & Poor & $\leq 3$ years & Begin Arranging Procedure to Adjust or Reconstruct Considering Risk \\
\hline $0-30$ & Very Poor & close to the end of life & Instantly Assess Risk \\
\hline
\end{tabular}

\subsection{0verall research workflow}

This research is divided into four major procedures. The main aim of these procedures is to obtain the transformer $\mathrm{HI}$ by applying ANN and scoring and ranking techniques. The findings of the transformer HI were compared to validate the proposed technique. The overall flowchart of this research is depicted in Figure 2.

\section{Results \& discussion}

This section discusses the findings of transformer $\mathrm{HI}$ prediction applying FFNN with LM, QNBP, and SCG as training algorithms. The findings were analyzed according to the MSE, R, and MAPE. This section also explains the findings of transformer $\mathrm{HI}$ applying the scoring and ranking method.

\subsection{Results of transformer HI applying LM} training algorithm

Table 5 shows the configuration of ANN with LM training algorithm. The number of hidden layers, momentum rate, and learning rate were set applying the heuristic method to find the best findings. The data consist of 160 transformer data with 112 training data and 48 testing data. The epoch and goal were set to 800 and $1 \times 10^{-15}$, respectively. From the findings, the values of MSE training and MSE testing were 1.5207 and 8.4705 , respectively. The values of MAPE were $0.000844 \%$ and less than $10 \%$ for accurate prediction. The value of $\mathrm{R}$ was 0.99717 .

The findings of predicted transformer HI applying LM training algorithm were compared to the calculation of ranking and scoring method. Table 6 shows the comparison of 20 transformers $\mathrm{HI}$ predicted applying the proposed method and the actual value obtained applying the scoring and ranking method. Figure 3 shows the comparison for all 160-transformer data. According to the findings, there was a slight difference between the ANN and the scoring and ranking method. Therefore, the proposed model of ANN is acceptable and can be used to predict the transformer HI. 
Nur Ashida Salim et al.

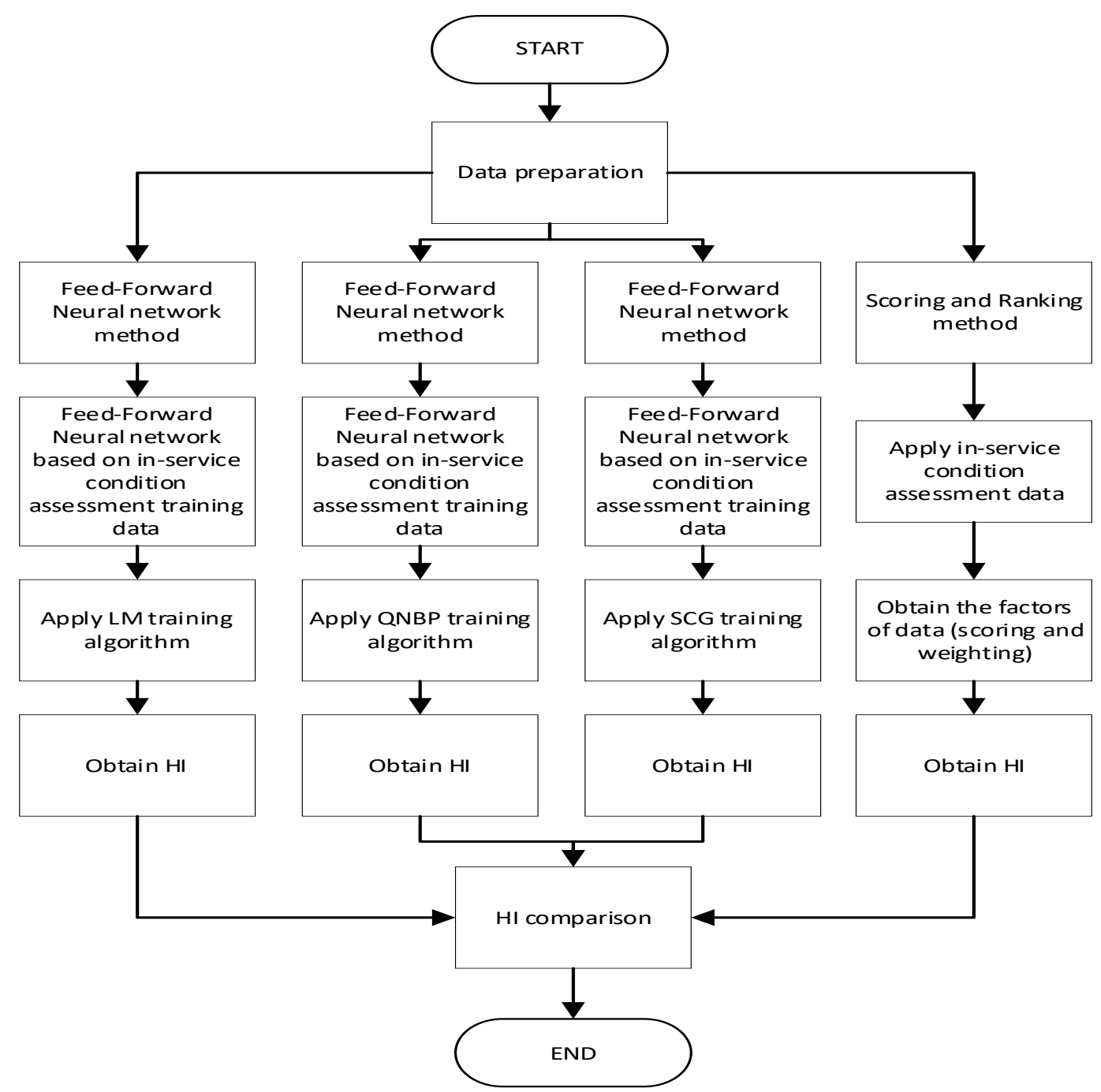

Figure 2 Overall flowchart of the research

Table 5 Raining algorithm of ANN configuration applying LM

\begin{tabular}{ll}
\hline Item & Model \\
\hline Network Configuration & 'Logsig', 'Purelin' \\
\hline Learning Rate & 0.2 \\
\hline Momentum Rate & 0.5 \\
\hline Number of Hidden Layer & 1 \\
\hline Training Technique & Levenberg-M arquadt (LM) \\
\hline Training Goal & $1 \times 10^{-15}$ \\
\hline Epoch & 800 \\
\hline No. Of Training Data & 112 \\
\hline No. Of Testing Data & 48 \\
\hline MSE Training & 1.5207 \\
\hline MSE Testing & 8.4705 \\
\hline Mean Absolute Percentage Error (MAPE) & $0.000844 \%$ \\
\hline R & 0.99717 \\
\hline Time (S) & $0: 00: 00$ \\
\hline
\end{tabular}


Table $6 \mathrm{HI}$ for ANN and scoring method

\begin{tabular}{lllll}
\hline \multirow{2}{*}{ No. of transformer } & \multicolumn{2}{c}{ Transformer health index $(\%)$} & & Transformer condition \\
\cline { 2 - 5 } & Scoring method & Transformer condition & FFNN- LM method & Very Poor \\
\hline 1 & 21.42857 & Very Poor & 20.65126 & Poor \\
\hline 3 & 33.33333 & Poor & 33.34193 & Very Good \\
\hline 4 & 88.09524 & Very Good & 91.94967 & Good \\
\hline 5 & 73.80952 & Good & 72.45868 & Good \\
\hline 6 & Good & 76.03481 & Very Poor \\
\hline 7 & 76.19048 & Very Poor & 25.01865 & Very Good \\
\hline 8 & 25.0000 & Very Good & 91.94967 & Good \\
\hline 10 & 88.09524 & Good & 76.03481 & Fair \\
\hline
\end{tabular}

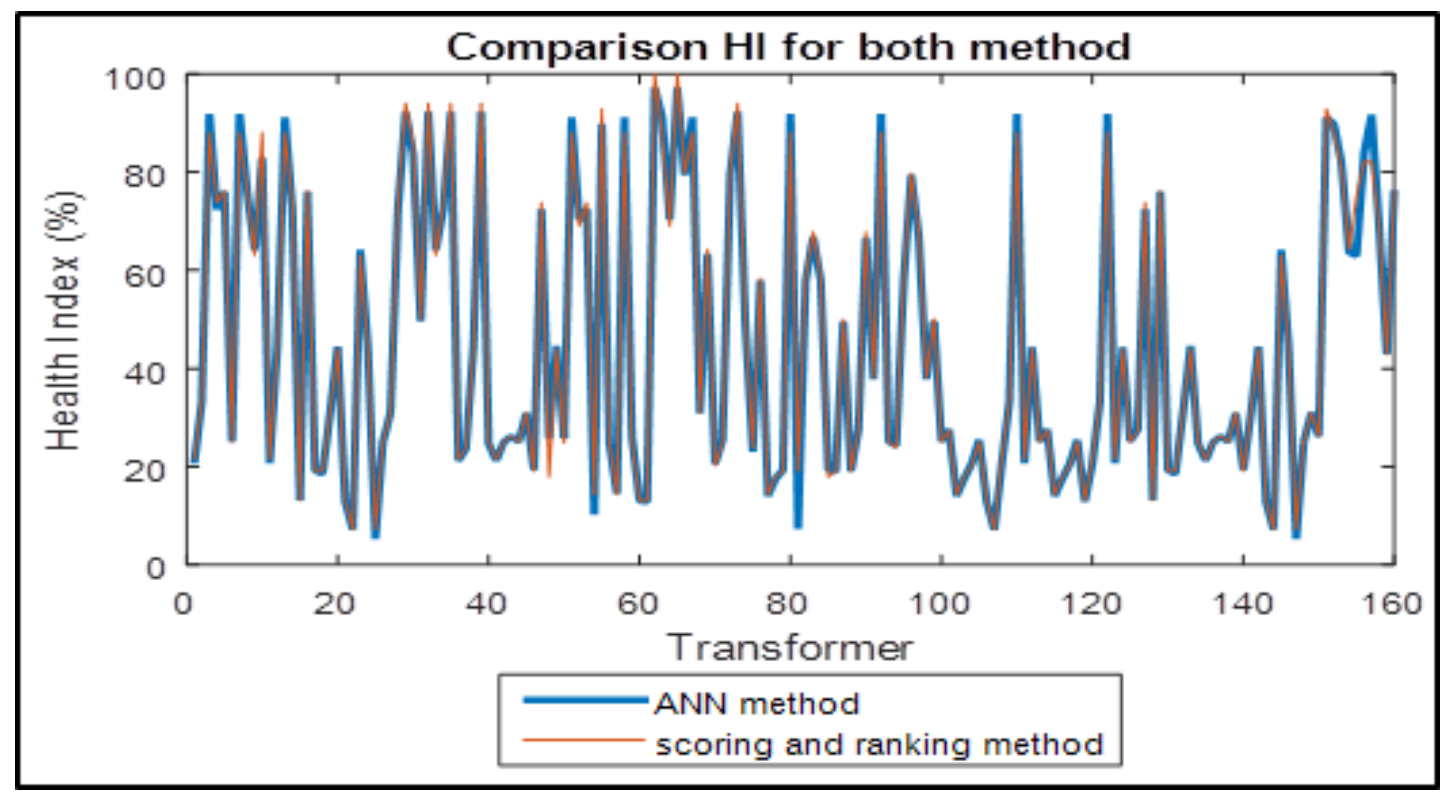

Figure 3 Comparis on of transformer HI between the proposed ANN method and scoring and ranking method

3.2Results of transformer HI applying QNBP training algorithm

Table 7 shows the configuration of the QNBP training algorithm. The training parameter was varied applying the heuristic method to obtain the best parameter. The total data of 160 transformer data were used and divided by the training-to-testing data ratio of 70:30. The learning rate, momentum rate, and hidden neuron were varied until a good value of MSE, R, and MAPE were obtained. The training goal and epochs were set to 0.001 and 5000, respectively. The time taken for the FFNN model according to QNBP method was 26 s. Table 8 shows the comparison of HI value between QNBP method and the scoring and ranking method for 10 transformers. The value of predicted HI was slightly different from the HI value of the scoring and ranking method.

Figure 4 shows the comparison of the HI value of 160 data between the FFNN method and the scoring and ranking method. The findings of the proposed model prediction were almost exactly similar to those of the scoring and ranking method. Thus, the model was validated, and it is accepted. 
Nur Ashida Salim et al.

Table 7 Configuration of QNBP

\begin{tabular}{ll}
\hline Item & Model \\
\hline Training Technique & Quasi-Newton Back Propagation (QNBP) \\
Network configuration & 'logsig', 'purelin' \\
Learning Rate & 0.2 \\
Momentum Rate & 0.5 \\
Number of Hidden Layer & 1 \\
Number of Hidden neurons & 10 \\
Training Goal & 0.001 \\
Epoch & 5000 \\
No. of Training Data & 112 \\
No. of Testing Data & 48 \\
MSE training & 0.00281 \\
MSE Testing & 0.00024 \\
MAPE & $0.00232 \%$ \\
R & 0.99621 \\
Time(s) & $0: 00: 26$ \\
\hline
\end{tabular}

Table 8 Comparis on Between FFNN-QNBP Model with Scoring and Ranking Method Transformer Health Index (\%)

\begin{tabular}{ccccc}
\hline $\begin{array}{l}\text { No.of } \\
\text { transformer }\end{array}$ & $\begin{array}{l}\text { Scoring } \\
\text { method }\end{array}$ & Transformer condition & FFNN-SCG method & Transformer condition \\
\hline 1 & 88.09524 & Very Good & 90.42863 & Very Good \\
\hline 2 & 73.80952 & Good & 73.20018 & Good \\
\hline 3 & 76.19048 & Good & 76.06048 & Good \\
\hline 4 & 73.80952 & Good & 73.20018 & Good \\
\hline 5 & 76.19048 & Good & 76.06048 & Good \\
\hline 6 & 19.04762 & Very Poor & 18.77911 & Very Poor \\
\hline 7 & 30.95238 & Poor & 30.35806 & Poor \\
\hline 8 & 63.09524 & Fair & 30.35806 & Fair \\
\hline 9 & 73.80952 & Good & 73.20018 & Good \\
\hline 10 & 94.04762 & Verv Good & 87.50267 & Verv Good \\
\hline
\end{tabular}

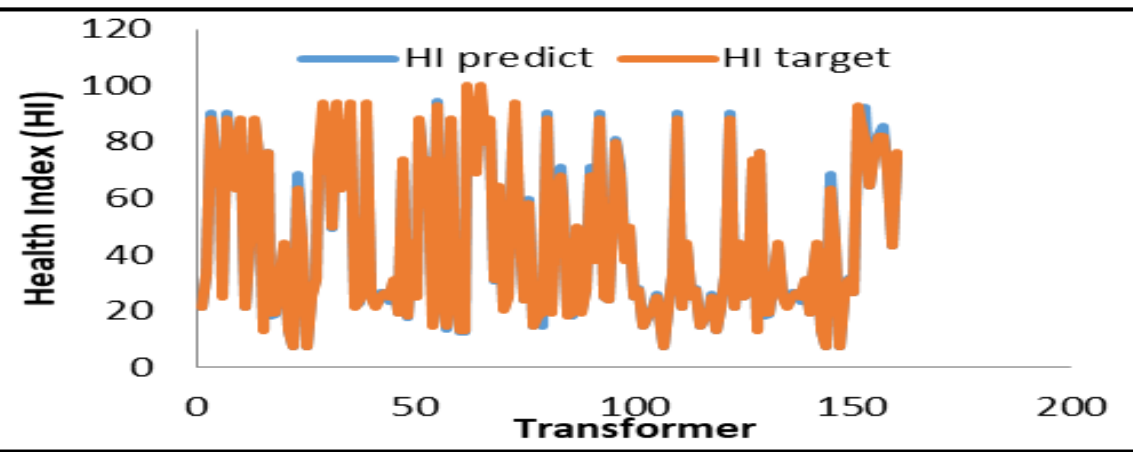

Figure 4 Comparison of HI value between proposed FFNN-QNBP with scoring and ranking method for overall transformer

3.3Results of transformer HI applying SCG training algorithm

Table 9 shows the configuration of the FFNN proposed in this research. The learning rate, momentum rate, and number of hidden layers were set according to the value as in Table 9. The data 300 comprises of total 160 data with 112 training data and 48 testing data. The goal and epoch were set to $1 \mathrm{e}-15$ and 2000 iterations, respectively. From the study, the values of MAPE were $0.00274 \%$ and less than $10 \%$, which indicate that the proposed model produced an accurate prediction. The values of MSE training and 
testing were 0.3707 and 10.6723 , respectively. The value of $\mathrm{R}$ was 0.99859 .

The findings of transformer HI obtained applying the SCG are shown in Table 10. The findings show that there was a slight difference between the two methods. Therefore, the FFNN method can be used to predict the transformer HI.

Table 9 Configuration of SCG propagation

\begin{tabular}{ll}
\hline Item & Model \\
\hline Network Configuration & 'logsig' 'purelin' \\
Learning rate & 0.2 \\
Momentum Rate & 0.5 \\
Number of Hidden Layer & 1 \\
Training Technique & Scale Conjugate Gradient (SCG) \\
Training goal & $1 \mathrm{e}-15$ \\
Epoch & 2000 \\
No. of Training Data & 112 \\
No. of Testing Data & 48 \\
MSE Training & 0.3707 \\
MSE Testing & 10.6723 \\
MAPE & $0.00274 \%$ \\
R & 0.99859 \\
Time(s) & $00: 00: 01$ \\
\hline
\end{tabular}

Table 10 HI Comparis on Between FFNN-SCG Model with scoring and ranking method

\begin{tabular}{lllll}
\hline \multicolumn{5}{l}{ Transformer health index (\%) } \\
\hline No. of transformer & Scoring method & $\begin{array}{l}\text { Transformer } \\
\text { condition }\end{array}$ & Ffnn- SCG method & Transformer condition \\
\hline 1 & 21.428 & Very Poor & 21.538 & Very Poor \\
2 & 33.333 & Poor & 32.995 & Poor \\
3 & 88.095 & Very Good & 88.323 & Very Good \\
\hline 4 & 73.809 & Good & 73.686 & Good \\
5 & 76.190 & Good & 76.529 & Good \\
6 & 25 & Very Poor & 24.728 & Very Poor Good \\
7 & 88.095 & Very Good & 88.323 & Fair \\
8 & 63.817 & Fair & 63.095 & Fair \\
9 & 63.095 & Fair & 63.817 & Very Good \\
\hline
\end{tabular}

\section{Conclusion}

This paper has presented the use of FFNN to predict the transformer $\mathrm{HI}$ and minimize the error by creating the best ANN model applying LM, QNBP, and SCG as the training algorithms. Since artificial intelligence is widely used around the world, it would be a great contribution to create a model that can predict the transformer HI. The findings show that all training algorithms were well implemented according to the MSE, R, and MAPE performance evaluation of the prediction. According to the transformer HI obtained, 301 preliminary actions can be taken by the power system utility whether to upgrade, perform maintenance, replacement, monitoring, repair, and contingency control of the transformer. This technique could assist the power system operation so that the lifespan of the transformer can be increased in any transmission condition. The proposed method of predicting the transformer health index could be further improved by developing an algorithm for accurate weighting factor in order to obtain the more reliable prediction in the future. 
Nur Ashida Salim et al.

\section{Acknowledgment}

The authors would like to thank the Research Management Institute (RMI), Universiti Teknologi MARA, Malaysia and the Ministry of Higher Education (MOHE), Malaysia through research grant 600 -IRMI/FRGS 5/3 (157/2019) for the financial support of this research.

\section{Conflicts of interest}

The authors have no conflicts of interest to declare.

\section{References}

[1] Jahromi A, Piercy R, Cress S, Service J, Fan W. An approach to power transformer asset management using health index. IEEE Electrical Insulation Magazine. 2009; 25(2):20-34.

[2] Raeisian L, Niazmand H, Ebrahimnia-Bajestan E, Werle P. Thermal management of a distribution transformer: an optimization study of the cooling system using CFD and response surface methodology. International Journal of Electrical Power \& Energy Sy stems. 2019; 104:443-55.

[3] Petchrompo S, Parlikad AK. A review of asset management literature on multi-asset systems. Reliability Engineering \& System Safety. 2019; 181:181-201.

[4] Ranga C, Chandel AK, Chandel R. Condition assessment of power transformers based on multiattributes using fuzzy logic. IET Science, Measurement \& Technology. 2017; 11(8):983-90.

[5] Pandey A, Sonwane PM. Implementation of reliability centred maintenance for transformer. In international conference on automatic control and dynamic optimization techniques 2016 (pp. 578-81). IEEE.

[6] Phadungthin R, Haema J. Risk management model of $69 \mathrm{kV}$ power transformer bushing in Metropolitan substation. In 4th international conference on industrial engineering and applications 2017 (pp. 3414). IEEE.

[7] Abu-Elanien AE, Salama MM, Ibrahim M. Determination of transformer health condition using artificial neural networks. In international symposium on innovations in intelligent systems and applications 2011 (pp. 1-5). IEEE

[8] Wattakapaiboon W, Pattanadech N. The new developed Health Index for transformer condition assessment. In international conference on condition monitoring and diagnosis (CMD) 2016 (pp. 32-5). IEEE.

[9] Ghazali YZ, Talib MA, Rosli HA. TNB experience in condition assessment and life management of distribution power transformers. In CIRED 2009-20th international conference and exhibition on electricity distribution-part 12009 (pp. 1-4). IET.

[10] Jasni J, Azmi A, Yahaya MS, Talib MA. Assessment of transformer health index using different model. Pertanika Journal of Science and Technology. 2017; 25:143-50.
[11] Scatiggio F, Pompili M. Health index: The TERNA's practical approach for transformers fleet management. In electrical insulation conference 2013 (pp. 178-82). IEEE.

[12] Ren M, Zeng W, Yang B, Urtasun R. Learning to reweight examples for robust deep learning. In international conference on machine learning 2018 (pp. 4334-43). PMLR.

[13] Wang SC. Artificial neural network. In interdisciplinary computing in java programming 2003 (pp. 81-100). Springer, Boston, MA.

[14] Shojaeefard MH, Zare J, Tabatabaei A, Mohammadbeigi $H$. Evaluating different types of artificial neural network structures for performance prediction of compact heat exchanger. Neural Computing and Applications. 2017; 28(12):3953-65.

[15] Wilamowski BM, Yu H. Improved computation for levenberg-marquardt training. IEEE Transactions on Neural Networks. 2010; 21(6):930-7.

[16] Nikov A, Stoeva S. Quick fuzzy backpropagation algorithm. Neural Networks. 2001; 14(2):231-44.

[17] Martens J. Deep learning via hessian-free optimization. In ICML 2010 (, pp. 735-42).

[18] Chaudhary P, Rizwan M. QNBP NN-based I $\cos \phi$ algorithm for PV systems integrated with LV/MV grid. Soft Computing. 2020:1-16.

[19] Kriegeskorte N. Deep neural networks: a new framework for modeling biological vision and brain information processing. Annual Review of Vision Science. 2015; 1:417-46.

[20] Cetişli B, Barkana A. Speeding up the scaled conjugate gradient algorithm and its application in neuro-fuzzy classifier training. Soft Computing. 2010; 14(4):365-78.

[21] Bakar NA, Abu-Siada A, Cui H, Li S. Improvement of DGA interpretation using scoring index method. In 1st international conference on electrical materials and power equipment 2017 (pp. 502-6). IEEE.

[22] Phadungthin R, Haema J. Development of risk optimization model for high voltage substation transformer maintenance. In PES Asia-pacific power and energy engineering conference (APPEEC) 2015 (pp. 1-5). IEEE.

[23] Hamrick L. Dissolved gas analysis for transformers. Neta World. 2009:1-4.

[24] Haema J, Phadungthin R. Development of condition evaluation for power transformer maintenance. In 4 th international conference on power engineering, energy and electrical drives 2013 (pp. 620-3). IEEE.

[25] Hernanda IN, Mulyana AC, Asfani DA, Negara IY, Fahmi D. Application of health index method for transformer condition assessment. In TENCON 20142014 IEEE Region 10 Conference 2014 (pp. 1-6). IEEE

[26] Islam MM, Lee G, Hettiwatte SN. A review of condition monitoring techniques and diagnostic tests for lifetime estimation of power transformers. Electrical Engineering. 2018; 100(2):581-605. 


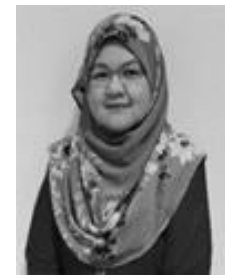

Nur Ashida Salim received her $\mathrm{PhD}$ in Electrical Engineering from Universiti Teknologi MARA in 2015, Master in Engineering (Power System \& Electrical Energy) from Universiti Malaya in 2006 and Bachelor in Electrical Engineering (Hons.) from Universiti Teknologi MARA in 2003. She is currently an Associate Professor at the Centre for Electrical Power Engineering Studies, Faculty of Electrical Engineering, Universiti Teknologi MARA. Her research includes Power System Reliability, Power System Planning, Power System Stability, Power System Asset Management and other related areas. To date, she has published more than 40 journal articles and many proceeding papers.

Email: nurashida606@uitm.edu.my

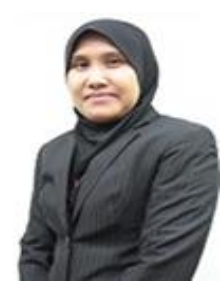

Jasronita Jasni received the B.Eng. degree in electrical engineering from Universiti Teknologi Malaysia, Johor, Malay sia, in 1998, the M.Eng. degree in electrical engineering from Universiti Teknologi Malaysia, Johor, Malaysia in 2001 and the Ph.D. degreee in electrical power engineering from Universiti Putra Malaysia, Selangor, Malaysia in 2010. From 1998-1999, she was a system engineer and later in 1999-2001, she worked as a tutor in Department of Electrical and Electronics Engineering, Faculty of Engineering, Universiti Putra Malaysia. Then, in 2001, she started working as a lecturer in the same department at Universiti Putra Malaysia. Currently, she is an associate professor at Department of Electrical and Electronics Engineering, Faculty of Engineering, Universiti Putra Malaysia. Her research interest includes Power System, Renewable Energy and Lightning Protection. She has been actively involved in Advanced Lightning, Power and Energy Research Centre (ALPER) as one of their key researchers.

Email: nurashida606@uitm.edu.my

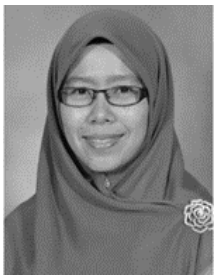

Hasmaini Mohamad received the B.Eng., M.Eng., and Ph.D. degrees from the University of Malaya, in 1999, 2004, and 2013, respectively. She started her career as a lecturer in Universiti Teknologi MARA in 2003 where currently she is an Associate Professor at the Centre for Electrical Power Engineering Studies, Faculty of Electrical Engineering. Apart from that, she has published more than 50 journal papers including high impact ISI journals and 20 conference papers. Her major research interest includes Islanding Operation of Distributed Generation, Hydro Generation, Load Sharing Technique, and Load Shedding Scheme.

Email: jas@upm.edu.my

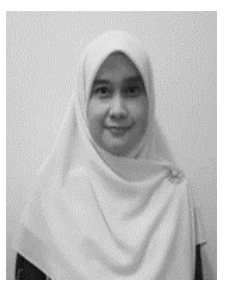

Zuhaila Mat Yasin graduated from Universiti Sains Malaysia with honours degree in Electrical and Electronics Engineering in 1998. She obtained her MSc degree in 2008 and $\mathrm{PhD}$ degree in 2015 from Universiti Teknologi MARA. She is currently a senior lecturer at Universiti Teknologi MARA. Her research interest includes Power System Operation, Optimization, Distributed Generation, Artificial Intelligence and Smart Grid System

Email: hasmaini@uitm.edu.my 